\title{
AUTOMATIC SEGMENTATION OF HUMAN IRIS IMAGES USING LOCALIZED ENERGY- BASED ACTIVE CONTOUR MODEL
}

\author{
P.Kalavathi and JMR.Bhonesh Narayani \\ Department of Computer Science and Applications \\ Gandhigram Rural Institute - Deemed University \\ Gandhigram, Tamil Nadu, India
}

\begin{abstract}
Biometric identification is one of the technologies used for automated personal identification. Iris recognition is biometric-based identification method which are more reliable and accurate authentication system. This paper present a simple and efficient method based on localized energy-based active contour model to segment iris from human eye image. First we segment the rough iris image and it is then redefined in the second level segmentation to produce better result. This proposed method is tested with the eye images obtained from CASIA database. The performance of the method is quantitatively evaluated by calculating the similarity measures Jaccard $(J)$ and Dice $(D)$. Experimental results show that the proposed method provides better segmentation result compared to the expert segmented images. .
\end{abstract}

\section{KEYWORDS}

Iris segmentation, Energy-based active contour method, Biometric authentication system, Iris recognition

\section{INTRODUCTION}

A traditional authentication such as password and/or PIN number became unsafe and insecure in the current scenario. Today's technology has introduced a much smarter, reliable, and secure solution called biometric authentication system. Biometric system provides reliable identification and verification of an individual and help in enhancing security infrastructure against some of the threats in the traditional authentication systems [1] [2]. Biometric identification are classified into two classes: the first class is called physiological which interested in the shape of the body like face, fingerprint, hand geometry and iris recognition. The second is called behavioural that are related to the behaviour of a person like signature and voice [3]. The iris recognition system is considered as one of the important ways of security in airport, military and research laboratories [4].

Among various biometric authentication methods, iris recognition is considered as a most reliable and accurate method because of its high recognition rate. In iris recognition system, iris is separated from the captured eye image and compared with the iris feature from the stored information [5]. Iris recognition has increased the popularity of the biometric system, and produced most reliable results [6]-[8]. A simple automatic iris segmentation method proposed in [9] [10] uses active contour method to segment iris in human eye image. Human authentication method using iris based on wavelet transform is proposed by Boles and Boashash [11]. Biometric recognition system proposed in [12] utilizes pattern recognition techniques on the basis of patterns obtained from iris, then results are compared with some analogy found in the literature. 
A method given in [13] uses a set of one-dimensional signals, extracted from iris image using the values of illumination intensity on a set of pupil and the centred circular contours which have been localized through use of edge detection techniques. The iris images are projected vertically and horizontally to estimate the centre of the iris. This method has also been utilized for eyelash segmentation and lightening reflection removal. Daugman [14] proposed an integral differential operator for proper segmentation of an iris. A segmentation approach based on initialization of mask's method is computationally expensive to detect the coordinates of the iris [15]. Kim [16] described a new iris recognition algorithm, which uses a low level of details, combining statistical classification, and elastic boundary fitting. In this method iris is first localized, and then the localized iris image is down-sampled by a factor of $\mathrm{m}$, and filtered by a modified laplacian kernel. Since the output of the laplacian operator is sensitive to a small shift of the full-resolution, therefore they are computed for all space shifts.

A novel fake iris detection method based on wavelet packet transform is developed in [17]. In this method wavelet packet decomposition is used to extract the attribute values which provide unique information for discriminating fake irises from the real ones. Then to enhance the detected accuracy of fake irises, support vector machine (SVM) is used to characterize the distribution boundary based on extracted wavelet packet features.

Couchant [18] developed a biometric system based on automatic identification of a person on a unique attribute or characteristic possessed by the individual. They used feature extraction for iris recognition process. The performance of this iris recognition system highly depends on edge detection, because sometimes even the effective feature extraction method would not able to obtain useful iris image feature [19]. In order to reduce the implementation time and increase the segmentation accuracy, a new iris segmentation, method based on localized energy-based active contour model is proposed in this paper. The remaining part of the paper is organized an follows; In section 2 the methodological details of the proposed method is given. The results and conclusions are given in section 3 and section 4 respectively.

\section{METHODOLOGY}

This proposed method uses a localized energy-based active contour model [9] for automatic segmentation of the iris in the eye image. The overall block diagram of the proposed method is given in Fig 1. This method first read the input image and convert it into gray scale image, if the input image is a colour image. The localized energy based active contour segmentation method need to define the initial mask for automatic segmentation. This method uses a automatic approach to define the initial mask based an width and height of the given input image. Usually the eye image contains not only the iris but also some outer part of eye like pupil, eyelids and sclera. The localization of iris is very difficult at the global segmentation, therefore in this proposed method we used localized energy based active contour method. Sometime, first level segmentation may include the other region of eye image, to avoid this, we have defined the initial mask in the rough segmented image and applied the same segmentation method for the second time. On each segmentation level, the local region-based energy framework is computed for guiding the active contour to evolve on the image border. 


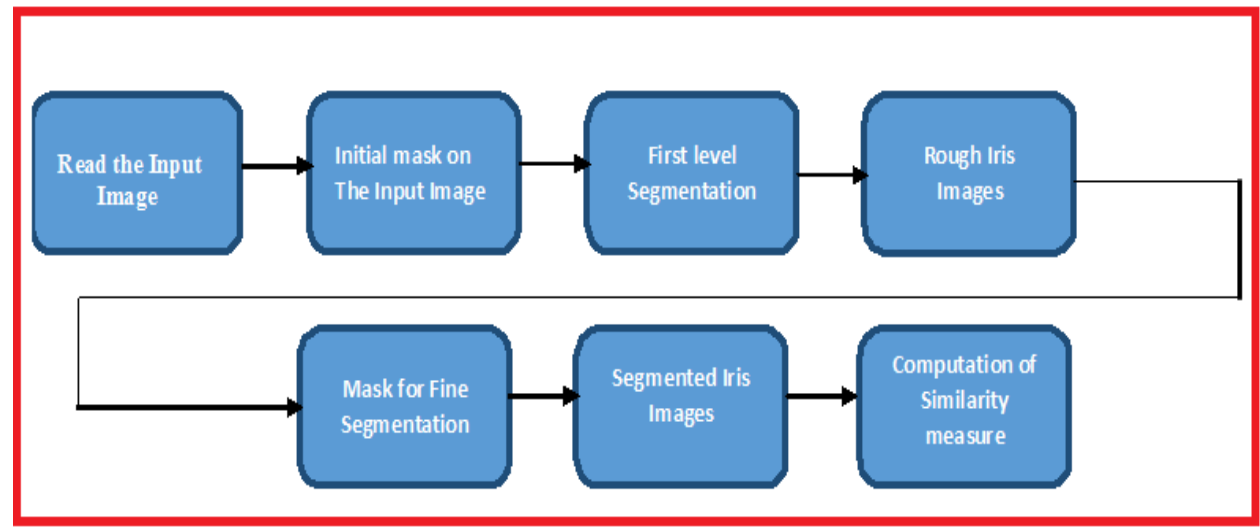

Fig.1: Block diagram of the proposed method

To compute these local energies, local neighbourhoods are split into local interior and local exterior by the evolving curve. In order to optimize the local energy on each localized region, each point is considered separately, and move to minimize (or maximize) the energy computed in its own local region. The energy optimization is then done by fitting a model to each local region.

Let I denote a given image on the domain $\Omega$, and let $\mathrm{C}$ be a closed contour represented as the zero level set of a signed distance function $\emptyset$, i.e., $C=\{x \mid \varnothing(x)=0\}$. It specify the interior of $\mathrm{C}$ by the following approximation of the smoothed Heaviside function:

$$
\phi(\mathrm{x})\left\{\begin{array}{lr}
1, & \phi(x)<-\epsilon \\
0, & \emptyset(x)>\epsilon \\
\frac{1}{2}\left\{1+\frac{\emptyset}{\mathrm{a}}+\frac{1}{\pi} \sin \left(\frac{\pi \phi(x)}{\pi}\right)\right\}, & \text { otherwise }
\end{array}\right.
$$

Similarly, the exterior of $\mathrm{C}$ is defined as $(1-\mathrm{H} \phi(\mathrm{x}))$. To specify the area just around the curve, it will use the derivative of $H \varnothing(x)$, a smoothed version of the Dirac delta.

$\delta \varnothing(x)\left\{\begin{array}{lr}1, & (x)=0 \\ 0, & |\Phi(x)|<\epsilon \\ \frac{1}{2 \epsilon}\left[1+\cos \left(\frac{\pi \varnothing(x)}{\varepsilon}\right)\right\}, & \text { otherwise }\end{array}\right.$

It now introduces a second spatial variable $\mathrm{y}$. This method uses $\mathrm{x}$ and $\mathrm{y}$ as independent spatial variables each representing a single point in $\Omega$. Using this notation, a characteristic function in terms of a radius parameter $r$ is defined.

$$
\mathrm{B}(\mathrm{x}, \mathrm{y})= \begin{cases}1, & \|x-y\|<r \\ 0, & \text { otherwise. }\end{cases}
$$

It use $\mathrm{B}(\mathrm{x}, \mathrm{y})$ to mask local regions. This function will be 1 when the point $\mathrm{y}$ is within a ball of radius $r$ centered at $x$, and 0 otherwise. 


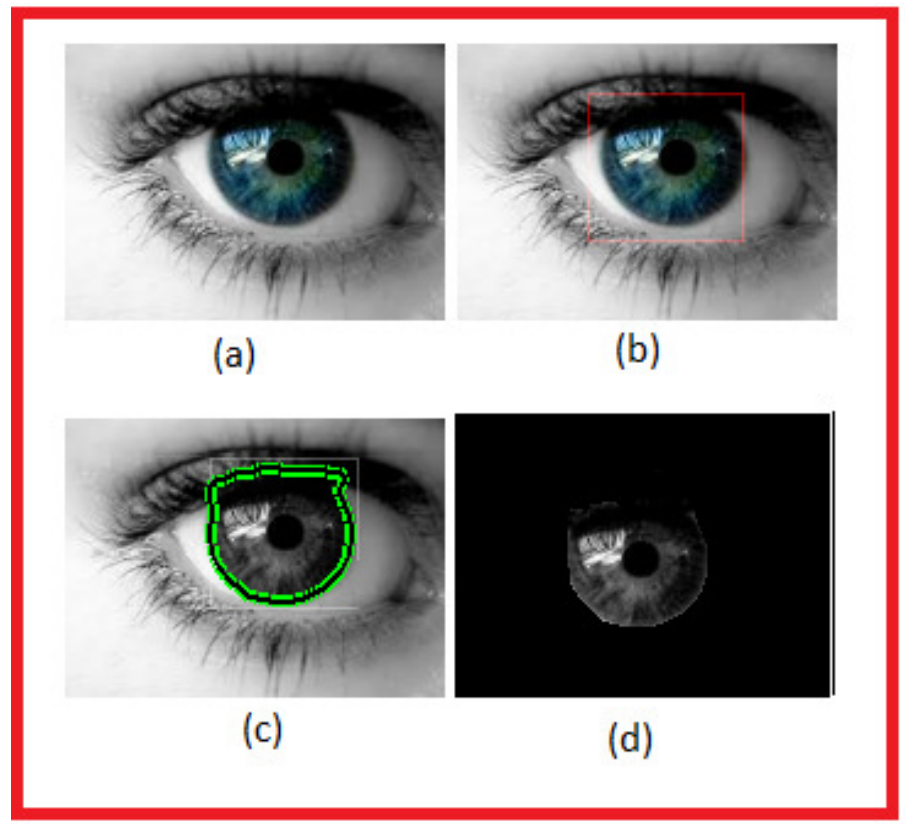

Fig 2: Process of Iris segmentation; (a) Original image (b) Defined initial mask

(c) Segmented curve (d) Segmented iris image

In Fig 2, the original input image in show in Fig 2(a), the automatic defined initial curve is given in Fig 2(b). The segmented curve is given in Fig 2(c), and final level segmented iris image is shown in Fig 2(d).

The summary of the steps involved in this proposed method is given below:

Step 1: Read the original image

Step 2: If the original image is colour image then convert it into gray scale image

Step 3: Define the initial mask on the eye image for the automatic segmentation of iris using localized energy-based active contour method

Step 4: Obtain the segmented iris image (ie) rough segmentation from the result of step 3

Step 5: Define the initial mask for the second level segmentation and segment the iris based on the same active contour method

Step 6: Obtain the final segmented iris image.

Step 7: Compute the similarity measure using the segmented iris image by the proposed and expert segmentation method.

\subsection{Performance Evaluation}

The performance of the proposed method is evaluated using the similarity measures Jaccard (J) and Dice (D). These methods will take two images as input and produce the value between 0 and 1 . 
The Jaccard [20] value is calculated by:

$$
\mathrm{J}(\mathrm{s} 1, \mathrm{~s} 2)=\frac{|S 1 \cap S 2|}{|S 1 \mathrm{~S} 2|}
$$

The Dice [20] value is calculated by:

$$
\mathrm{D}(\mathrm{s} 1, \mathrm{~s} 2)=\frac{2|s 1 \mathrm{n} s 2|}{|s 1|+|s 2|}
$$

where, $\mathrm{S} 1$ represents the total pixels of the image obtained by the proposed segmentation method and $\mathrm{S} 2$ represents the total pixels in image obtained from ground truth image.

\subsection{Datasets Used}

To evaluate the performance of this proposed method, we used eye images from the dataset [21], the Chinese Academy of Science-Institute of Automation (CASIA) eye image dataset containing 256 gray scale eye images with 108 unique eye or classes and 7 different images of each unique eye. Images from each class are taken from two sessions with one month interval between the sessions. The images were captured especially for iris recognition research using specialized digital optics developed by the National Laboratory of Pattern Recognition, China. The eye images are mainly from persons of Asian descent, whose eyes are characterized by irises that are densely pigmented, and with dark eyelashes. Due to specialized imaging conditions using near infrared light, features in the iris region are highly visible and there is good contrast between pupil, iris and sclera regions.

\section{RESULTS AND DISCUSSION}

In this method, we used images from CASIA to evaluate the performance of the proposed method. A quantitative evaluation also done by computing the similarity measures Jaccard (J) and Dice (D) as per the equation given in Eq (4) and (5). A selected sample image from the eye dataset along with the segmentation result by the proposed and expert method are shown in Fig. 3.

In Fig.3, the original eye image is given in column (a) and the segmented iris by the proposed and the expert methods are shown in Fig 3(b) and Fig 3(c) respectively. We have also computed similarity measures Jaccard (J) and Dice (D) for quantitative result. The computed J and D value for the image show in Fig. 3 are given in Table 1. It is evident from Fig. 3 and table 1 is that the proposed localized energy-based active contour method efficiently segmented the iris from the eye image.

Table 1: Computed Jaccard (J) and Dice Similarity Measure for the image shown in Fig. 3.

\begin{tabular}{|c|c|c|c|}
\hline S.No & Images & Jaccard values (J) & Dice values (D) \\
\hline 1 & Image 1 & 0.7620 & 0.8012 \\
\hline 2 & Image 2 & 0.8250 & 0.8746 \\
\hline 3 & Image 3 & 0.7634 & 0.8487 \\
\hline 4 & Image 4 & 0.7039 & 0.7476 \\
\hline 5 & Image 5 & 0.8295 & 0.8543 \\
\hline 6 & Image 6 & 0.7566 & 0.8725 \\
\hline 7 & Image 7 & 0.6389 & 0.7689 \\
\hline 8 & Image 8 & 0.7295 & 0.8561 \\
\hline 9 & Image 9 & 0.7290 & 0.7953 \\
\hline 10 & Image10 & 0.8543 & 0.9000 \\
\hline
\end{tabular}




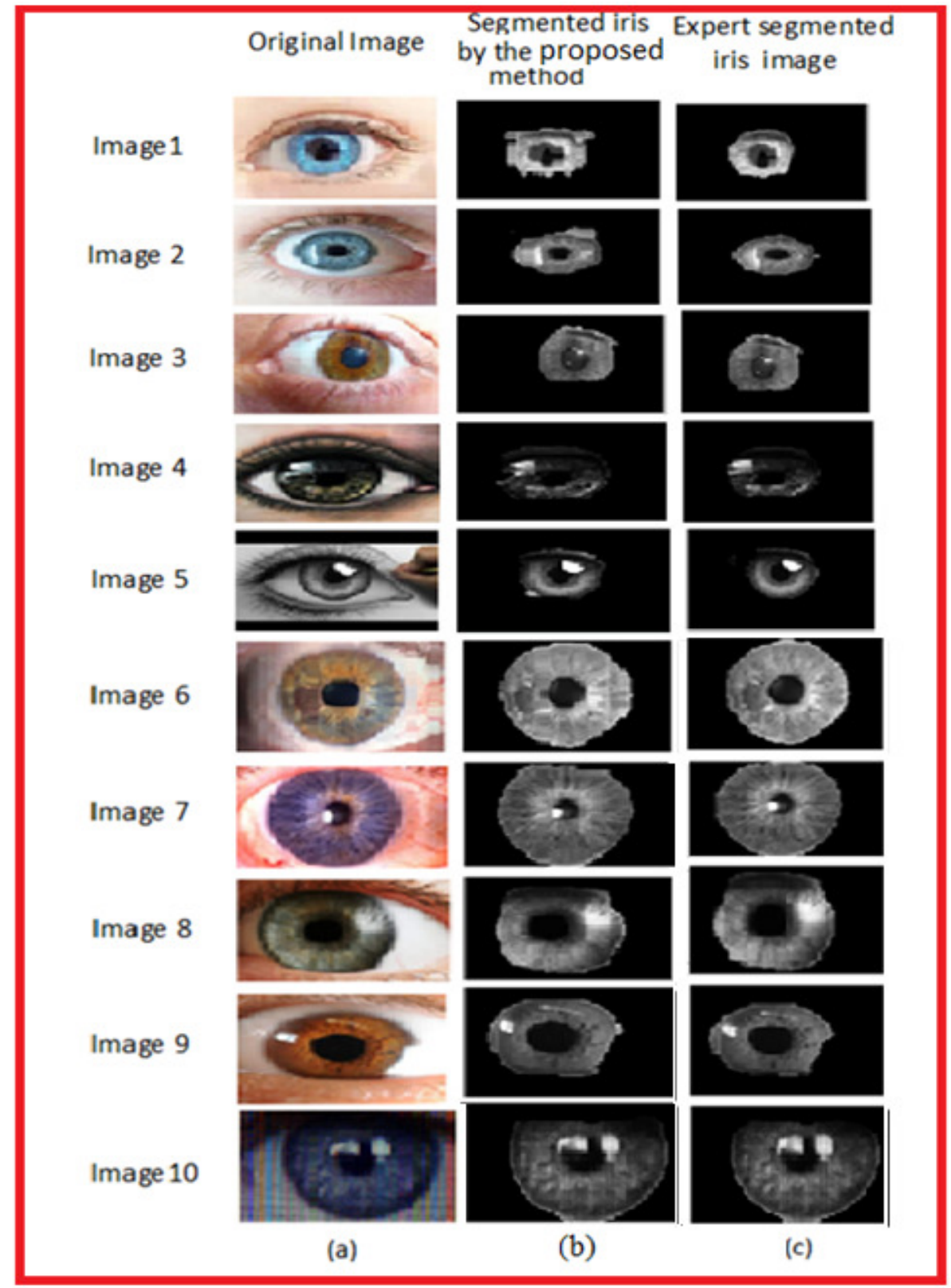

Fig 3: Iris Segmentation result by the proposed method; (a) Original images (b) Segmented iris by the proposed method (c) Expert segmented iris image

\section{CONCLUSIONS}

A simple method to segment the iris from eye image is proposed in this paper. we used localized energy-based active contour method to isolate iris from eye images. We applied bi-level segmentation approach which could efficiently remove eyelid and eyelash from the eye image. For quantitative analysis, the Jaccard (J) and Dice (D) similarity measure have been calculated based on the expert segmented result. The experimental results show that the proposed method has an acceptable performance. 


\section{REFERENCES}

[1] Rozeha A. Rashid, Nur Hija Mahalin, Mohd Adib Sarijari, Ahmad Aizuddin Abdul Aziz, "Security system using biometric technology. Design and implementation of voice recognition system (VRS)", International conference on computer and communication Engineering Kuala Jumpur, Malaysia, Vol 23, pp.898-90, 2008.

[2] Daugman, "New methods in iris recognition and segmentation", IEEE Trans, on system, man and cybernetics, Vol. 37, no.10, pp. 1167-1175, 2007.

[3] S. Liz and B. Stephen, "A human identification technology using images of the iris and wavelet transform," IEEE Trans on signal processing, Vol. 46, no. 4, pp. 1185-1188, 1998.

[4] R. Wides, "Iris recognition; an emerging biometric technology", proceedings of the IEEE, vol.85, no 9, pp.1348-1363, 1997.

[5] Prateek Verma, Maheedhar Dubey, Praveen Verma, "Comparison of varios segmentation techniques in iris recognition" LAMBERT ACADEMIC PUBLISHING (LAP), GmbH and co. KG, Dudweiler Landstrab, Saarbrucken, ISBN 13: Vol. 46, no. 3, pp. 281-307, 2012.

[6] Robert C. Schultz, Robert Welvet,"Biometric data acquisition using MATLAB GUIs", $35^{\text {th }}$ ASEE/IEEE for iris identification", IEEE proceedings, Vol. 34, no. 5, pp.1207-1210, 2005.

[7] Chen, Wen-Shiung and Yuan, Shang-Yuan," An automatic iris. Recognition system based on fractal dimension", VIP-CcLab., Department of Electrical Engineering, National chian university., Vol. 53, no. 2, pp. 195-205, 2006.

[8] CO.H.Seal, M.M Gifford and D.J. McCartney, "Iris recognition for user validation", IEEE transactions on pattern analysis and machine intelligence., Vol. 23, no. 7, pp. 61-70, 2001.

[9] Shawn Lankton, "Localizing Region-Based Active Contours", IEEE Trans., on image processing Vol. 17, no. 11, pp, 234-567, 2008.

[10] P. Kalavathi and JMR. Bhonesh Narayani,"Automatic segmentation and recognition of human iris images" New horizons in computational intelligence and information systems, Excel India publishers, India, 2015.

[11] W. Boles and B. Boasbasb, "A human initialization technique using authenticate themselves by entering a secret password and PIN", IEEE Trans. Signal processing, vol.46, no. 4, pp.1185-1188, 1998

[12] Xiao he, "A new fake iris detection method using wavelet packet transform", Department of computer science and technology, East china normal university, OSpringer-verlag Berlin heidel berg Vol. 40, no. 19,pp.379-467, 2010.

[13] Bhawna Chouhan, Shailja Shukla. "Iris recognition system using canny edge detection for biometric identification", International Journal of Engineering Sciences and Technology (IJEST), ISSN: Vol.3, no. I. pp.0975-5462, 2011.

[14] Daugman, J., "How iris recognition works", IEEE Transactions on circuits and systems for video technology, Vol. 14, no. 1, pp. 21-30, 2004.

[15] Muhammana Kharram Khan, Joshua Zhang, Shi-Jinn Horng, "An effective iris automatic segmentation system for identification of humans", proceedings of IEEE,Vol. 33, no. 15, pp.114-117. 2004.

[16] Jaemin Kim, "Iris recognition using a Low of details", School of Electronics and Electrical Engineering, Hongik University, 72-I Sangsu-Dong, Seoul, no. 1, pp. 121-791, Korea, 2006

[17] Paul,"Support vector machine (SVM) is used to characterize the distribution boundary based on Extracted wavelet packet feature. Vol .24, no. 9, pp. 453-0892, 2008

[18] Couchant, "Biometric system based on automatic segmentation and identification" IEEE Trans., on single processing, Vol. 46. no.4, pp.1185-1188.1998

[19] Richard Yen Fattyng, Kai Ming Mok, "Iris biometric recognition for person identification in security system”- International Journal of Computer Applications Vol. 24, no. 9, pp. 0975-8887 ,2011

[20] K. Somasundaram and P. Kalavathi, A novel skull stripping technique for T1-weighted MRI human head scans, ACM digital Library 1pp.1-8, 2012.

[21] CASIA iris database. Institute of Automation, Chinese Academy of Sciences http://www. Cbsr.ia.ac.cn \Iris Database. Html 


\section{Authors}

Dr.P.KALAVATHI, Associate Professor in Computer Science and Applications, Gandhigram Rural Institute - Deemed University, obtained her B.C.A degree from Mother Teresa Women's University, Kodaikanal; Post-Graduate Degree in Computer Applications (M.C.A) from GRI-DU; M.Phil, in Computer Science from Bharathidasan University, Tiruchirappalli; and Doctoral Degree in Computer Science and Applications from GRI-DU, Gandhigram. She has qualified UGC-NET for Lectureship in 2000. She has 15 years of Teaching and 8 years of Research experience. The author has more than 50 research articles in various journals and edited volumes. Her research area focuses on Digital Image Processing and Medical Image Segmentation \& Analysis. She is also serving as a reviewer for many international conferences and for various journals in IEEE, Springer, Elsevier etc. The author is the Life Member of Indian Society for Technical Education (ISTE), New Delhi.

JMR.BHONESH NARAYANI,Completed Bachelor of Science in Information Technology in Madurai Sivakasi Nadar Pioneer Meenakshi Women's College, Alagappa University, and Master of Science in Information Technology in Gandhigram Rural Institute-Deemed University, Tamil Nadu. She is currently pursuing M.Phil (Computer Science) in Gandhigram Rural Institute-Deemed University, Tamil Nadu. She has published one research article in National Conference proceedings. Her research area focus on Biometric image processing especially iris segmentation and recognition. 